\title{
A GENERALIZATION OF CENTRALIZER NEAR-RINGS
}

\author{
by KIRBY C. SMITH
}

(Received 24th April 1984)

\section{Introduction}

Let $G$ be a group with identity 0 and let $\mathscr{A}$ be a group of automorphisms of $G$. The centralizer near-ring determined by $G$ and $\mathscr{A}$ is the set $C(\mathscr{A} ; G)=\{f: G \rightarrow G \mid f \alpha=\alpha f$ for all $\alpha \in \mathscr{A}$ and $f(0)=0\}$, forming a near-ring under function addition and function composition. This class of near-rings has been extensively studied (for example see [1], [2], [5] and [6]) and it is known that every finite simple near-ring with identity which is not a ring is isomorphic to $C(\mathscr{A} ; G)$ for a suitable pair $(\mathscr{A}, G)$ see [6] page 131 , Corollary 4.59 and Theorem 4.60 .

As illustrated in [1] a key to the study of the near-ring $C(\mathscr{A} ; G)$ is the orbit structure of $G$ determined by $\mathscr{A}$. For each $v \in G$ the stabilizer of $v$ is $\operatorname{stab}(v)=\{\alpha \in \mathscr{A} \mid \alpha v=v\}$, a subgroup of $\mathscr{A}$. If $v, w \in G$ belong to the same $\mathscr{A}$-orbit then there exists a $\beta \in \mathscr{A}$ such that $w=\beta v$ and we have $\operatorname{stab}(w)=\beta \operatorname{stab}(v) \beta^{-1}$. So two elements of $G$ from the same orbit have conjugate stabilizers.

Definition 1. Let $G$ be a group and $\mathscr{A}$ a group of automorphisms of $G$. We will call the pair $(\mathscr{A}, G)$ normal if

(a) $G$ has finitely many $\mathscr{A}$-orbits, and

(b) if $v, w \in G$ belong to the same $\mathscr{A}$-orbit and if $\operatorname{stab}(v) \subseteq \operatorname{stab}(w)$ then $\operatorname{stab}(v)=$ $\operatorname{stab}(w)$.

We remark that if $G$ is a finite group then $(\mathscr{A}, G)$ is normal. Also we note that (b) is the finiteness condition used by Meldrum and Zeller in [5].

Although near-rings of the form $C(\mathscr{A} ; G)$ are of fundamental importance in the theory of near-rings, it is difficult to decide whether or not a given near-ring is isomorphic to $C(\mathscr{A} ; G)$ for some group $G$ and group of automorphisms $\mathscr{A}$ ([2], [4]). In this paper a class $\mathcal{N}$ of near-rings is defined which contains all centralizer near-rings of the form $C(\mathscr{A} ; G)$ where $(\mathscr{A}, G)$ is normal. It will be shown that many of the results on centralizer near-rings are true for the near-rings in $\mathscr{N}$. Moreover to decide whether or not a nearring belongs to $\mathscr{N}$ is in general easier than deciding whether or not it is a centralizer near-ring $C(\mathscr{A} ; G)$.

\section{Generalized centralizer near-rings}

Let $N$ be a near-ring with identity 1 . An element $e \in N$ is idempotent if $e \neq 0$ and $e^{2}=e$. If $e_{i}$ and $e_{j}$ are idempotents in $N$ we will let $N_{i j}$ denote the set $N_{i j}=e_{i} N e_{j}=$ 
$\left\{e_{i} n e_{j} \mid n \in N\right\}$, a subset of $N$. The idempotents $e_{i}$ and $e_{j}$ are orthogonal if $e_{i} e_{j}=e_{j} e_{i}=0$. Finally an idempotent $e \in N$ is primitive if $e$ is not the sum of two orthogonal idempotents in $N$.

Definition 2. Let $N$ be a zero symmetric near-ring with 1 then $N$ is a $G C$ near-ring (generalized centralizer near-ring) if the following six axioms are satisfied.

(i) There is a finite number of idempotents $e_{1}, \ldots, e_{s} \in N$ such that $1=e_{1}+\cdots+e_{s}$, $e_{i} e_{j}=0$ for all $i, j$ with $i \neq j$ and $e_{i}+e_{j}=e_{j}+e_{i}$ for all $i, j$.

(ii) For $i=1, \ldots, s$ the set $\left(e_{i} N e_{i}\right)^{*}=N_{i i}^{*} \equiv N_{i i}-\{0\}$ is a group under multiplication having identity $e_{i}$.

(iii) Let $n_{i_{1} j_{1}} \in N_{i_{1} j_{1}}, \ldots, n_{i_{j} j_{s}} \in N_{i_{s} j_{s}}$ with $\left\{j_{1}, \ldots, j_{s}\right\}=\{1, \ldots, s\}$, then for every $f \in N$, $f\left(n_{i_{1} j_{1}}+\cdots+n_{i_{s} j_{s}}\right)=f n_{i_{1} j_{1}}+\cdots+f n_{i_{s} j_{s}}$.

(iv) For every $f \in N$ and $n_{k j} \in N_{k j}$ then $f n_{k j}$ belongs to $N_{t j}$ for some $t$ ( $t$ depends on $f$ and $n_{k j}$ ).

(v) For every $n_{i j} \in N_{i j}$ and $n_{k j} \in N_{k j}$ then $n_{i j}+n_{k j} \in N_{t j}$ for some $t$ ( $t$ depends on $n_{i j}$ and $n_{k j}$ ).

(vi) If $N_{i j} \neq\{0\}$ and $N_{j k} \neq\{0\}$ then $N_{i j} N_{j k} \neq\{0\}$.

For convenience we make the convention that $N=\{0\}$ is a $G C$ near-ring.

Proposition 1. Let $N$ be a GC near-ring using idempotents $e_{1}, \ldots, e_{s}$. Then each $e_{i}$ is a primitive idempotent.

Proof. Suppose $e_{i}=f_{1}+f_{2}$ where $f_{1}$ and $f_{2}$ are idempotents such that $f_{1} f_{2}=f_{2} f_{1}=0$. We have $e_{i} f_{1}=\left(f_{1}+f_{2}\right) f_{1}=f_{1}^{2}+f_{2} f_{1}=f_{1}$. Similarly $e_{i} f_{2}=f_{2}$. Also $\left(f_{1} e_{i}\right)^{2}=f_{1} e_{i} f_{1} e_{i}=$ $f_{1} f_{1} e_{i}=f_{1} e_{i}$, an element of $N_{i i}$. By axiom (ii) either $f_{1} e_{i}=e_{i}$ or $f_{1} e_{i}=0$. Since $0 \neq f_{1}=$ $f_{1}^{2}=e_{i} f_{1} e_{i} f_{1}$ then $f_{1} e_{i} \neq 0$, so $f_{1} e_{i}=e_{i}$. A similar argument shows $f_{2} e_{i}=e_{i}$. But then $e_{i}=e_{i}^{2}=\left(f_{1}+f_{2}\right) e_{i}=f_{1} e_{i}+f_{2} e_{i}=e_{i}+e_{i}$ which implies $e_{i}=0$, a contradiction. So $e_{i}$ is primitive.

Our first main result implies that the set of idempotents $\left\{e_{1}, \ldots, e_{s}\right\}$ in axiom (i) for a $G C$ near-ring is unique.

Theorem 1. Suppose $N$ is a near-ring satisfying axioms (i)-(v) using idempotents in the set $E=\left\{e_{1}, \ldots, e_{s}\right\}$, and $N$ also satisfies axioms (i)-(v) using idempotents in the set $F=\left\{f_{1}, \ldots, f_{t}\right\}$, then $E=F$.

Proof. For $k=1, \ldots, t$ we have $f_{k}=f_{k}\left(e_{1}+\cdots+e_{s}\right) f_{k}=f_{k} e_{1} f_{k}+\cdots+f_{k} e_{s} f_{k}$ using axioms (i) and (iii). Since $f_{k} \neq 0$ then $f_{k} e_{j} f_{k} \neq 0$ for some $j$. By axiom (iv) there is an $i$ such that $e_{j} f_{k} \in N_{i k}^{\prime} \equiv f_{i} N f_{k}$, and since $f_{k} e_{j} f_{k} \neq 0$ then $e_{j} f_{k} \in N_{k k}^{\prime}$, that is $i=k$. We now have $f_{k} e_{j} f_{k}=e_{j} f_{k}$. The element $e_{j} f_{k}$ is idempotent since $\left(e_{j} f_{k}\right)^{2}=e_{j} f_{k} e_{j} f_{k}=e_{j} e_{j} f_{k}=e_{j} f_{k}$. Since $e_{j} f_{k} \in N_{k k}^{\prime}$ axiom (ii) implies $e_{j} f_{k}=f_{k}$.

We have now shown that for each $k, k=1, \ldots, t$, there is a $j$, depending on $k$, such that $f_{k} e_{j} f_{k}=e_{j} f_{k}=f_{k}$. Moreover this $j$ is unique, for if $f_{k} e_{i} f_{k}=e_{i} f_{k}=f_{k}$ then $f_{k}=e_{j} f_{k}=e_{i} f_{k}$ and 
$i=j$. Similarly for each $j, j=1, \ldots, s$, there exists a unique $c=c(j)$ such that $e_{j} f_{c} e_{j}=$ $f_{c} e_{j}=e_{j}$. Hence $s=t$. The maps are universes of each other since $f_{k}=f_{k} e_{j} f_{k}=f_{k} f_{c} e_{j} f_{k}$ implies $k=c$. We may reorder the $e_{i}$ 's if necessary so that $1=f_{1}+\cdots+f_{s}=e_{1}+\cdots+e_{s}$ with $e_{i} f_{i}=f_{i}$ and $f_{i} e_{i}=e_{i}$. For $i=1, \ldots, s$ we have $f_{i}=f_{i}\left(e_{1}+\cdots+e_{s}\right)=f_{i} f_{1} e_{1}+\cdots+f_{i} f_{s} e_{s}=$ $f_{i} f_{i} e_{i}=e_{i}$. This shows $E=F$.

Proposition 2. Let $N$ be a GC near-ring with respect to the set of idempotents $E=\left\{e_{1}, \ldots, e_{s}\right\}$. Suppose $I$ is an ideal of $N$ which is a near-ring with identity $f$. Then $f=e_{i_{1}}+\cdots+e_{i_{t}}$ where $\left\{e_{i_{1}}, \ldots, e_{i_{t}}\right\} \subseteq E$.

Proof. Suppose that $f e_{i} \neq 0$ where $e_{i} \in E$. Then $f e_{i} \in I$ since $I$ is an ideal. Also $\left(f e_{i}\right)^{2}=$ $f e_{i} f e_{i}=f e_{i} e_{i}=f e_{i}$ using the fact that $f$ is the identity on $I$. By axiom (iv), $f e_{i}$ belongs to $N_{k i}$ for some $k$. Since $f e_{i}$ is idempotent then $k=i$ and $f e_{i}=e_{i}$ using axiom (ii). So if $f e_{i} \neq 0$ then $f e_{i}=e_{i} \in I$. Let $e_{i_{1}}, \ldots, e_{i_{i}}$ be the idempotents in $E$ such that $f e_{i_{j}} \neq 0$. We have $f=f\left(e_{1}+\cdots+e_{s}\right)=f e_{1}+\cdots+f e_{s}=e_{i_{1}}+\cdots+e_{i_{f}}$.

The following theorem indicates that the class $\mathcal{N}$ of $G C$ near-rings is a "large" class with nice properties.

Theorem 2. Let $\mathcal{N}$ be the class of GC near-rings. The following are properties of $\mathcal{N}$.

(a) $\mathscr{N}$ contains all near-rings of the form $C(\mathscr{A} ; G)$ where $(\mathscr{A}, G)$ is normal.

(b) If $N \in \mathcal{N}$ and $I$ is an ideal of $N$ which is a near-ring with identity then $I \in \mathcal{N}$.

(c) If $N_{1}, N_{2} \in \mathcal{N}$ then $N_{1} \oplus N_{2} \in \mathcal{N}$.

(d) If $N \in \mathscr{N}$ and if $I$ is an ideal of $N$ such that $I \in \mathcal{N}$, then $N / I \in \mathcal{N}$.

Proof. (a) Suppose $N=C(\mathscr{A} ; G)$ with nonzero $\mathscr{A}$-orbits $\theta_{1}, \ldots, \theta_{s}$. For $i=1, \ldots, s$ let $e_{i}: G \rightarrow G$ be the function which is the identity on orbit $\theta_{i}$ and zero elsewhere. Then $e_{i} \in N$ and $e_{i}$ is idempotent. We have $1=e_{1}+\cdots+e_{s}$ and it is straightforward to check that axioms (i)-(vi) are satisfied.

(b) Let $N$ satisfy axioms (i)-(vi) using idempotents in the set $E=\left\{e_{1}, \ldots, e_{s}\right\}$. By Proposition 2 the identity element $f$ of $I$ may be written $f=e_{i_{1}}+\cdots+e_{i_{i}}$ where $F=\left\{e_{i_{1}}, \ldots, e_{i_{t}}\right\}$ is a subset of $E$. The near-ring $I$ is a $G C$ near-ring using $F$.

(c) Since $N_{1} \in \mathscr{N}$ there are idempotents $e_{1}, \ldots, e_{s} \in N_{1}$ such that axioms (i)-(vi) are true. Similarly for $N_{2}$ using idempotents $e_{1}^{\prime}, \ldots, e_{t}^{\prime}$. In $N_{1} \oplus N_{2}$ we have $1=e_{1}+\cdots+$ $e_{s}+e_{1}^{\prime}+\cdots+e_{t}^{\prime}$ and axioms (i)-(vi) are easily verified.

(d) If $N$ satisfies axioms (i)-(vi) using idempotents in $E=\left\{e_{1}, \ldots, e_{s}\right\}$ then Proposition 2 implies $I$ is a $G C$ near-ring using $F=\left\{e_{i_{1}}, \ldots, e_{i_{t}}\right\} \subseteq E$. Without loss of generality we may assume $F=\left\{e_{1}, \ldots, e_{t}\right\}$. We claim that $N / I$ is a $G C$ near-ring using idempotents $\bar{E}=\left\{\bar{e}_{t+1}, \ldots, \bar{e}_{s}\right\}$ where $\bar{e}_{i}=e_{i}+I$. Axioms (i) $-(\mathrm{v})$ are obviously true. To check axiom (vi) suppose $\bar{N}_{i j} \neq\{0\}$ and $\bar{N}_{j k} \neq\{\overline{0}\}$ where $\bar{N}_{i j}=\bar{e}_{i} \bar{N} \bar{e}_{j}$, etc. In $N$ we have $N_{i j} N_{j k} \neq\{0\}$ by axiom (vi). Since $\{0\} \neq N_{i j} N_{j k} \subseteq N_{i k}$ and $N_{i k} \cap I=\{0\}$ then $\bar{N}_{i j} \bar{N}_{j k} \neq\{\overline{0}\}$ as desired.

Theorem 3. A GC near-ring $N$ is a ring if and only if $N$ is a (finite) direct sum of division rings. 
Proof. Suppose $N$ is a ring which is also a $G C$ near-ring using idempotents $e_{1}, \ldots, e_{s}$. We will show that if $i \neq j$ then $N_{i j}=\{0\}$. Assume by way of contradiction that $N_{i j} \neq\{0\}$ and select a nonzero element $n_{i j} \in N_{i j}$. By axiom (v) $n_{i j}+e_{j}$ belongs to $N_{i j}$ for some $t$. We have $n_{i j}+e_{j}=e_{t}\left(n_{i j}+e_{j}\right)=e_{t} n_{i j}+e_{t} e_{j}$ since $N$ is a ring. If $t=i$ then $n_{i j}+e_{j}=n_{i j}$ and $e_{j}=0$, impossible. If $t=j$ then $n_{i j}+e_{j}=e_{j}$ and $n_{i j}=0$, a contradiction. Finally, if $t \neq i, j$ then $n_{i j}+e_{j}=0$ and $n_{i j}=-e_{j} \in N_{j j}$ which is not possible since $N_{i j} \cap N_{j j}=\{0\}$. Hence $N_{i j}=\{0\}$ whenever $i \neq j$ and using axiom (ii), $N$ is a direct sum of division rings.

It is clear that a division ring is a $G C$ near-ring and by Theorem $2 \mathscr{N}$ contains all finite direct sums of division rings.

We note that $\mathscr{N}$ is a larger class of near-rings than the class of centralizer near-rings $C(\mathscr{A} ; G)$ with $(\mathscr{A}, G)$ normal, for it was shown in [4] that not every direct sum of fields is a centralizer near-ring $C(\mathscr{A} ; G)$.

It is trivial to verify that every nonzero homomorphic image of a near-ring $N$ satisfying axioms (i)-(v) also satisfies axioms (i)-(v). However, a nonzero homomorphic image of a $G C$ near-ring need not be a $G C$ near-ring as the following example shows. Thus the hypotheses in Theorem 2, part (d) are necessary.

Example. Let $G=Z_{8}$, the additive group of integers modulo 8. Let $\mathscr{A}=$ $\left\{\alpha_{1}, \alpha_{3}, \alpha_{5}, \alpha_{7} \mid \alpha_{i}: G \rightarrow G\right.$ defined by $\left.\alpha_{i}(a)=i a\right\}$, a group of automorphisms of $G$. Finally let $N=C(\mathscr{A} ; G)$. The nonzero $\mathscr{A}$-orbits of $G$ are $\theta(1)=\{1,3,5,7\}, \theta(2)=\{2,6\}$, and $\theta(4)=\{4\}$. The set $I=\{f \in N \mid f(1) \in\{0,4\}, f(2)=0, f(4)=0\}$ is an ideal of $N$.

We claim that $\bar{N}=N / I$ is not a $G C$ near-ring. Let $e_{1}, e_{2}, e_{4}$ be the idempotents in $N$ that are the identity on $\theta(1), \theta(2), \theta(4)$ respectively and zero elsewhere. Then $N$ is a $G C$ near-ring using $e_{1}, e_{2}, e_{4}$ and $\bar{N}$ satisfies axioms (i)-(v) using $\bar{e}_{1}, \bar{e}_{2}, \bar{e}_{4}$. By Theorem 1 $\left\{\bar{e}_{1}, \bar{e}_{2}, \bar{e}_{4}\right\}$ is the only set of idempotents for which $\bar{N}$ satisfies axioms (i)-(v). It suffices now to show that $\bar{N}$ does not satisfy (vi) using $\left\{\bar{e}_{1}, \bar{e}_{2}, \bar{e}_{4}\right\}$. Let $n_{42} \in N_{42} \equiv e_{4} N e_{2}$ and $n_{21} \in N_{21} \equiv e_{2} N e_{1}$, then $n_{42} n_{21} \in I$ so $N_{42} N_{21} \subseteq I$. Also $N_{42} \cap I=\{0\}$ and $N_{21} \cap I=\{0\}$. In $\bar{N}$ we have $\bar{N}_{42}=\bar{e}_{4} N \bar{e}_{2} \neq\{\overline{0}\}, \bar{N}_{21} \neq\{\overline{0}\}$ and yet $\bar{N}_{42} \bar{N}_{21}=\{\overline{0}\}$, violating axiom (vi).

\section{Structure theorems}

In this section we investigate the structure of $G C$ near-rings. In particular we determine when a $G C$ near-ring $N$ is simple and when it is semi-simple. Moreover we present a decomposition theorem for left ideals in $N$. These results generalize results known for centralizer near-rings ([1], [5], [6] and [7]).

Theorem 4. A GC near-ring $N$ is simple if and only if $N_{i j} \neq\{0\}$ for all $i$ and $j$.

Proof. Assume $N_{i j} \neq\{0\}$ for all $i, j$ where $N$ is a $G C$ near-ring using $E=\left\{e_{1}, \ldots, e_{s}\right\}$. Let $I$ be a nonzero ideal of $N$ and select $g \neq 0$ in $I$. Using axioms (i) and (iii) we have $g=\left(e_{1}+\cdots+e_{s}\right) g\left(e_{1}+\cdots+e_{s}\right)=\sum e_{j} g e_{i}$. Since $g \neq 0$ then $e_{j} g e_{i} \neq 0$ for some $j, i$. Also $e_{j} g e_{i} \equiv n_{j i} \in I \cap N_{j i}$. By axiom (vi) $N_{i j} N_{j i} \neq\{0\}$ so there exists $m_{i j} \in N_{i j}$ such that $0 \neq m_{i j} n_{j i} \in I \cap N_{i i}$. This means $e_{i} \in I$. For each $k$ and for each $n_{k i} \in N_{k i}$ we have $n_{k i} e_{i}=n_{k i} \in I \cap N_{k i}$. As above this implies $e_{k} \in I$. So $e_{1}+\cdots+e_{s}=1 \in I$ and $I=N$. 
For the converse assume $N_{i j}=\{0\}$ for some $i, j$ where $i \neq j$. Let $I=\operatorname{Ann}\left(N e_{j}\right)$, the annihilator of $N e_{j}$. The set $I$ is a left ideal of $N$ since it is the annihilator of a set of elements in $N$. Since $N \cdot N e_{j} \subseteq N e_{j}, I$ is an ideal of $N$. We have $I \neq\{0\}$ since $e_{i} \in I$ and $I \neq N$ since $e_{j} \notin I$. So $N$ is not simple.

Theorem 5. A GC near-ring $N$ is $J_{2}$-semisimple if and only if whenever $N_{i j}=\{0\}$ then $N_{j i}=\{0\}$.

Proof. Assume $N$ is semisimple. Suppose $N_{i j}=\{0\}$ but $N_{j i} \neq\{0\}$. Select a nonzero element $n_{j i} \in N_{j i}$ and let $M=N n_{j i}$, an $N$-subgroup of $N$. For $g \in N$ we have $g n_{j i} \in M \cap N_{k i}$ for some $k$ using axiom (iv). We claim the product of any two elements in $M$ is 0 . Clearly $\left(f n_{j i}\right)\left(g n_{j i}\right)=0$ if $k \neq i$. If $k=i$ then $g n_{j i}=e_{i} g n_{j i} e_{i}=e_{i} g e_{j} n_{j i} e_{i}$ and $e_{i} g e_{j}=0$ since $N_{i j}=$ $\{0\}$. Hence $g n_{j i}=0$ when $k=i$. This shows $M$ is nilpotent and $N$ is not semisimple since the $J_{2}$-radical of $N$ contains all nilpotent $N$-subgroups ([6], page 153 ).

For the converse we may assume $N_{i j}=\{0\}$ for some $i \neq j$. (For if $N_{i j} \neq\{0\}$ for all $i, j$ then $N$ is simple by Theorem 4.) Let $S_{j}=\left\{k \mid N_{k j} \neq\{0\}\right\}$, and if $S_{j}=\left(k_{1}, \ldots, k_{t}\right\}$ let $I=$ $N e_{k_{1}}+\cdots+N e_{k_{t}}$, a left ideal of $N$. We claim that $I$ is an ideal of $N$. To prove the claim it suffices to prove that $\left(n_{1} e_{k_{1}}+\cdots+n_{t} e_{k_{t}}\right) n_{k l} \in I$ for all $n_{1}, \ldots, n_{t} \in N$ and all $k, l$. If $\left(n_{1} e_{k_{1}}+\cdots+n_{t} e_{k_{l}}\right) n_{k l} \neq 0$ then $k \in S_{j}$ and $n_{k l} \neq 0$. Since $n_{k l} \neq 0$ then $N_{k l} \neq\{0\}$ and $N_{l k} \neq\{0\}$. So $N_{l k} N_{k j} \neq[0\}$ and $l \in S_{j}$. Since $l \in S_{j}, I$ contains every element in $N_{i l}, i=1,2, \ldots, s$ and $I$ contains $\left(n_{1} e_{k_{1}}+\cdots+n_{t} e_{k_{t}}\right) n_{k l}=n_{1} e_{k_{1}} n_{k l}+\cdots+n_{t} e_{k_{t}} n_{k l}$. Hence $I$ is an ideal of $N$.

Let $\bar{S}_{j}=\{1, \ldots, s\}-S_{j}$ and let $\bar{I}=N e_{i_{1}}+\cdots+N e_{i_{v}}$ where $\bar{S}_{j}=\left\{i_{1}, \ldots, i_{v}\right\}$. The set $\bar{I}$ is a left ideal of $N$ and we want to show it is an ideal. As before if $\left(n_{1} e_{i}+\cdots+n_{v} e_{i}\right) n_{k l} \neq 0$ then $k \in \bar{S}_{j}$ and $n_{k l} \neq 0$. If $l \in S_{j}$ then $N_{l j} \neq\{0\}$ and $\{0\} \neq N_{k l} N_{l j} \subseteq N_{k j}$. But $N_{k j} \neq\{0\}$ implies $k \in S_{j}$, a contradiction. Hence $l \in \bar{S}_{j}$ and $\left(n_{1} e_{i_{1}}+\cdots+n_{v} e_{i_{v}}\right) n_{k l} \in \bar{I}$. So $\bar{I}$ is an ideal of $N$ with $I \cap I=\{0\}$. If $\bar{I}$ is not a simple near-ring the above process may be repeated. Ultimately we obtain $N$ as a direct sum of finitely many simple near-rings, and $N$ is semisimple.

We next establish a decomposition theorem for left ideals in a $G C$ near-ring. This theorem is established with the aid of two propositions which are similar to results found in [7] for centralizer near-rings. The proofs are different and seem less technical than those for centralizer near-rings. In what follows $N$ is a $G C$ near-ring using idempotents $e_{1}, \ldots, e_{s}$.

Proposition 3. Let $L$ be a left ideal of a GC near-ring $N$. If $f \in L$ with $f e_{j} \in N_{k j}, k \neq j$, and if $f e_{j}+e_{j} \in N_{k j}$ then $e_{j} \in L$.

Proof. We have $e_{k} f e_{j}=f e_{j}$ and $e_{k} f e_{j}+e_{j}=f e_{j}+e_{j} \in N_{k j}$. Since $e_{k} f \in L$ we may assume $e_{k} f=f$. Using the left ideal property of $L$ we have $e_{k}\left(f+e_{j}\right)-e_{k} e_{j}=e_{k}\left(f+e_{j}\right) \in L$. Let $g=e_{k}\left(f+e_{j}\right)$ then $g e_{j}=e_{k}\left(f e_{j}+e_{j}\right)=f e_{j}+e_{j}$ since $f e_{j}+e_{j} \in N_{k j}$. If $i \neq j$ then $g e_{i}=e_{k} f e_{i}=f e_{i}$. We have $-f+g \in L$ and $-f+g=(-f+g)\left(e_{1}+\cdots+e_{s}\right)=\left(-f e_{1}+g e_{1}\right)+\cdots+$ $\left(-f e_{s}+g e_{s}\right)=0+\cdots+\left(-f e_{j}+f e_{j}+e_{j}\right)+\cdots+0=e_{j}$.

Proposition 4. Let $L$ be a left ideal of a GC near-ring $N$. Suppose $f \in L$ such that $e_{j} f e_{i} \neq 0$ and there exists an $m_{i j} \in N_{i j}$ with $m_{i j} e_{j} f e_{i}=e_{i}$, then $e_{i} \in L$. 
Proof. Let $n_{j i}=e_{j} f e_{i}$, then $m_{i j} f \in L, e_{i}\left(m_{i j} f\right)=m_{i j} f$, and $m_{i j} f e_{i}=m_{i j} e_{j} f e_{i}=e_{i}$. So we may assume $i=j$ and that $f \in L$ is such that $e_{i} f=f$ and $f e_{i}=e_{i}$. We may also assume $f e_{k} \neq 0$ for some $k \neq i$ or else $f=e_{i} \in L$ and we are done.

Among those $f \in L$ with $e_{i} f=f, f e_{i}=e_{i}$ select one such that the number of $k$ with $f e_{k} \neq 0$ is minimal. Now let $k$ be such that $k \neq i$ and $f e_{k} \neq 0$.

Case 1. Suppose there exists a $g \in N$ such that $e_{i} g e_{k}=0$ and $f e_{k}+g e_{k} \notin N_{i k}$. Let $h=e_{i}\left(f+g e_{k}\right)-e_{i} g e_{k}=e_{i}\left(f+g e_{k}\right)$. We have $h \in L, h e_{i}=e_{i}$ and $e_{i} h=h$. Moreover if $j \neq k$ then $h e_{j}=f e_{j}$ and $h e_{k}=0$. This contradicts the minimality of $f$.

Case 2. Assume $f e_{k}+g e_{k} \in N_{i k}$ for all $g$ such that $e_{i} g e_{k}=0$. Let $g=e_{k}$. Then $f \in L, f e_{k} \in N_{i k}$ and $f e_{k}+g e_{k}=f e_{k}+e_{k} \in N_{i k}$. By Proposition 3, $e_{k} \in L$. This means $f-f e_{k} \equiv h \in L$. We have $h e_{i}=\left(f-f e_{k}\right) e_{i}=f e_{i}=e_{i}$. Also $e_{i} h=e_{i}\left(f-f e_{k}\right)\left(e_{1}+\cdots+e_{k}+\right.$ $\left.\cdots+e_{s}\right)=e_{i}\left(f e_{1}-0\right)+\cdots+e_{i}\left(f e_{k}-f e_{k}\right)+\cdots+e_{i}\left(f e_{s}-0\right)=e_{i} f e_{1}+\cdots+0+\cdots+e_{i} f e_{s}=f e_{1}+$ $\cdots+0+\cdots+f e_{s}$, and $h=h\left(e_{1}+\cdots+e_{k}+\cdots+e_{s}\right)=h e_{1}+\cdots+h e_{k}+\cdots+h e_{s}=f e_{1}+$ $\cdots+0+\cdots+f e_{s}$. This shows $e_{i} h=h$. We also have $h e_{k}=0$ and this contradicts the minimality of $f$. So $e_{i} \in L$ as desired.

Theorem 6. Let $L$ be a left ideal of $N$, where $N$ is a GC near-ring using idempotents $e_{1}, \ldots, e_{s}$. Then for each $i, L e_{i} \subseteq L$. Also $L=L e_{1}+\cdots+L e_{s}$.

Proof. For the first part we need to show that if $f \in L$ then $f e_{i} \in L, i=1, \ldots, s$. We have $f e_{i} \in N_{k i}$ for some $k$ and so $e_{k} f e_{i}=f e_{i}$. This shows we may assume $e_{k} f=f$. If $f e_{i}=f$ we are done so we may assume $f e_{j} \neq 0$ for some $j \neq i$. If we can show that $f-f e_{j} \in L$ then we have $f^{(1)} \equiv f-f e_{j} \in L$ with $f_{1}^{(1)} e_{i}=f e_{i}$ and $f^{(1)} e_{j}=0$. So $f^{(1)}$ has one fewer $j$ such that $f^{(1)} e_{j} \neq 0$. This process can be continued until we have $f^{(t)} \in L$ such that $f^{(t)} e_{i}$ $=f e_{i}$ and $f^{(t)} e_{j}=0$ for all $j \neq i$. Then $f e_{i}=f^{(t)} e_{i}=f^{(t)}\left(e_{1}+\cdots+e_{s}\right)=f^{(t)} \in L$.

So it remains to show that $f-f e_{j} \in L$. If $j=k$ then $0 \neq e_{k} f e_{j}=e_{k} f e_{k} \in N_{k k}$ and Proposition 4 applies. So $e_{j}=e_{k} \in L$ and $f-f e_{j} \in L$.

If $j \neq k$ we have two cases to consider.

Case 1. Assume $e_{k}\left(f+e_{j}\right) e_{j} \neq 0$. By axiom (v) we have $\left(f+e_{j}\right) e_{j}=f e_{j}+e_{j} \in N_{t j}$ for some $t$. We must have $t=k$ and so $e_{k}\left(f+e_{j}\right) e_{j}=\left(f+e_{j}\right) e_{j}$. Let $g=e_{k}\left(f+e_{j}\right)-e_{k} e_{j}=e_{k}\left(f+e_{j}\right)$, an element of $L$. Note that $g e_{j}=e_{k}\left(f+e_{j}\right) e_{j}=\left(f+e_{j}\right) e_{j}=f e_{j}+e_{j}$ and if $l \neq j$ then $g e_{l}=$ $f e_{l}$. We have $-f+g \in L$ and $(-f+g)=(-f+g)\left(e_{1}+\cdots+e_{s}\right)=-f e_{j}+f e_{j}+e_{j}=e_{j} \in L$. So $f-f e_{j} \in L$ as desired.

Case 2. Assume $e_{k}\left(f+e_{j}\right) e_{j}=0$. Let $g=e_{k}\left(f+e_{j}\right)-e_{k} e_{j}=e_{k}\left(f+e_{j}\right)$, an element in $L$. We have $g e_{t}=f e_{t}$ for $t \neq j$ and $g e_{j}=0$. Using axiom (i), $g=g\left(e_{1}+\cdots+e_{j}+\cdots+e_{s}\right)=$ $g e_{1}+\cdots+g e_{j-1}+g e_{j+1}+\cdots+g e_{s}=f e_{1}+\cdots+f e_{j-1}+f e_{j+1}+\cdots+f e_{s}+f e_{j}-f e_{j}=f\left(e_{1}+\right.$ $\left.\cdots+e_{j-1}+e_{j+1}+\cdots+e_{s}+e_{j}\right)-f e_{j}=f-f e_{j}$, an element in $L$.

We have now shown that $f e_{i} \in L$ for every $f \in L$. It remains to prove that $L=L e_{1} \oplus \cdots \oplus L e_{s}$. We note that $L e_{i}$ is a left ideal of $N$ since $L e_{i}=$ $L \cap A n n\left\{e_{1}, \ldots, e_{i-1}, e_{i+1}, \ldots, e_{n}\right\}$ and that $L e_{i} \cap L e_{j}=\{0\}$ if $i \neq j$ since $L e_{i} \cdot e_{i}=L e_{i}$ and $L e_{j} \cdot e_{i}=\{0\}$. Since $L e_{i} \subseteq L$ for each $i$, then $L e_{1}+\cdots+L e_{s} \subseteq L$. On the other hand if $g \in L$ then $g=g\left(e_{1}+\cdots+e_{s}\right)=g e_{1}+\cdots+g e_{s}$ which implies $L \subseteq L e_{1}+\cdots+L e_{s}$. So $L=L e_{1} \oplus \cdots \oplus L e_{s}$.

Our final theorem gives more information about left ideals in a $G C$ near-ring and has relevance to Theorem 2 . 
Theorem 7. Suppose $N$ is a GC near-ring using idempotents $e_{1}, \ldots, e_{s}$. The following are equivalent.

(a) $N$ contains no nonzero nilpotent left ideals.

(b) $N e_{i}$ is a minimal left ideal for each $i$.

(c) Every nonzero left ideal of $N$ is generated by an idempotent.

(d) If $L$ is a nonzero left ideal then there exist idempotents $e_{i_{1}}, \ldots, e_{i_{k}}$ such that $L=N e_{i_{1}} \oplus \cdots \oplus N e_{i_{k}}$.

Proof. We will prove $a \Rightarrow b \Rightarrow d \Rightarrow c \Rightarrow a$.

$\mathrm{a} \Rightarrow$ b. Assume $N e_{i}$ is not minimal, say $\{0\} \neq L \subset N e_{i}$ where $L \neq N e_{i}$. Then $L=L e_{i}$ by Theorem 6, and if $l \in L$ then $l=l e_{i} \in N_{k i}$ for some $k$ where $N_{i k}=\{0\}$. So if $l_{1}, l_{2} \in L$ then $l_{1} l_{2}=0$ and $L$ is nilpotent, a contradiction. So $N e_{i}$ is minimal.

$\mathrm{b} \Rightarrow \mathrm{d}$. Let $L$ be a nonzero left ideal of $N$. Then $L=L e_{1} \oplus \cdots \oplus L e_{n}$ where $L e_{i}$ is a left ideal of $N$ contained in $N e_{i}$. Since $N e_{i}$ is minimal then either $L e_{i}=N e_{i}$ or $L e_{i}=\{0\}$.

$\mathrm{d} \Rightarrow \mathrm{c}$. If $L$ is a nonzero left ideal of $N$ then $L=N e_{i_{1}} \oplus \cdots \oplus N e_{i_{k}}=N\left(e_{i_{1}}+\cdots+e_{i_{k}}\right)$.

$c \Rightarrow a$. Obvious.

If $N$ is a $G C$ near-ring satisfying any of the conditions a-d in Theorem 7 then every ideal of $N$ and every homomorphic image of $N$ is a $G C$ near-ring.

Acknowledgements. The author thanks the referee for pointing out that the finiteness condition (part (b) of Definition 1) is needed in Theorem 2 part (a). Also the referee pointed out simplifications in several proofs, especially the proof of Theorem 1.

\section{REFERENCES}

1. C. J. Maxson, and K. C. SMith, The centralizer of a set of group automorphisms, Comm. in Algebra 8 (1980), 211-230.

2. C. J. Maxson, and K. C. Sмiтh, Centralizer near-ring representations, Proc. Edin. Math. Soc. 25 (1982), 145-153.

3. C. J. Maxson, and K. C. Smith, Centralizer near-rings: left ideals and 0-primitivity, Proc. Royal Irish Acad. 81A (1981), 187-199.

4. C. J. Maxson, M. R. Pettet and K. C. Smith, On semisimple rings that are centralizer nearrings, Pacific J. of Math. 101 (1982), 451-461.

5. J. D. P. Meldrum and M. Zeller, Simplicity of near-rings of mappings, Proc. Roy. Soc. Edinburgh 90A (1981), 185-193.

6. G. PILz, Near-rings, revised edition (North-Holland, 1983).

7. K. C. SMith, The lattice of left ideals in a centralizer near-ring is distributive, Proc. Amer. Math. Soc. 85 (1982), 313-317.

Department of Mathematics

TEXAs A\&M UNIVERSITY

College Station

TEXAS 77843-3368 